\title{
The Robust Output Tracking Problem for a Class of Discrete-time Linear Systems
}

\author{
Omar Zakary \\ Laboratory of Analysis Modeling \\ and Simulation, Department of \\ Mathematics and Computer Science, \\ Faculty of Sciences Ben M'Sik, \\ Hassan II University of Casablanca, \\ BP 7955, Sidi Othman, \\ Casablanca, Morocco
}

\author{
Mostafa Rachik \\ Laboratory of Analysis Modeling \\ and Simulation, Department of \\ Mathematics and Computer Science, \\ Faculty of Sciences Ben M'Sik, \\ Hassan II University of Casablanca, \\ BP 7955, Sidi Othman, \\ Casablanca, Morocco
}

\author{
Samih Lazaiz \\ Laboratory of Algebra Analysis \\ and Applications, Department of \\ Mathematics and Computer Science, \\ Faculty of Sciences Ben M'Sik, \\ Hassan II University of Casablanca, \\ BP 7955, Sidi Othman, \\ Casablanca, Morocco
}

\begin{abstract}
The robust tracking and model following problem of linear discrete-time systems is investigated in this paper. An approach to design a robust tracking controllers for this class of linear systems is proposed. First, it is assumed that system states must be fully accessible. The system is controlled to track dynamic outputs generated by a reference model. By using the the Lyapunov stability, the convergence of the tracking error to the origin, is proved. An application to a class of disturbed systems is considered. Numerical examples are given to demonstrate the validity of our results. Second, it is assumed that the system states are not accessibles. An observer is designed firstly, and then based on the observed states the controller is designed. The proposed approach employs linear controllers rather than nonlinear ones. Therefore, the designing method is simple for use and the resulting controller is easy to implement.
\end{abstract}

\section{General Terms}

Robust tracking, observer based control

\section{Keywords}

Robust tracking, model following, discrete-time systems, disturbances, observer

\section{INTRODUCTION}

During the last two decades, the robust tracking and model following problem have made much progress. Linear state feedback controllers are employed for robust tracking of dynamical systems [1] 2, 3, 4] and references therein. In [2], the authors presented a linear robust tracking controller for a class of uncertain time-delay systems. By using a Riccati-type equation, in [5] the researchers develop an improved procedure for determining the controller such that larger uncertainties are accommodated. While the proposed scheme of [2] is based on the solution of the Lyapunov equation. In [6] the study requires that the dimension of the reference model be the same as the dimension of the nominal systems under con- sideration. This presents a major limitation in the design of model reference controllers. In some instances, one may require a highorder system to follow a low-order reference model. In [7] this assumption is dropped and the dimension of the reference model is allowed to be unequal to the dimension of the nominal system under consideration. Practical tracking is achieved when the tracking error can be made arbitrarily small. In [8] and [9] the authors developed nonlinear robust controllers to achieve practical tracking for a class of uncertain systems. In the case when there is no control over the tracking error bound, the system is said to $\epsilon$-track the input. Authors of [1] developed a linear controller to achieve practical tracking for matched uncertainties and $\epsilon$-tracking for mismatched uncertainties when certain conditions are satisfied.

The tracking error is guaranteed to decrease asymptotically to zero, or asymptotic tracking is achieved in [10, 11]. Similar to these works, for a class of unconstrained linear discrete-time systems, this paper further investigates the problem of robust tracking and model following. By using a Lyapunov-type equation, we propose a new approach to the design of linear robust tracking and model following controllers, that ensures the convergence of the tracking error to the origin. Furthermore, there is no conditions on the dimension of the reference model.

In the most control systems, the existence of disturbances has a remarkable probability. The influence of the physical environment on the systems leads to the emergence of these undesirable parameters [12, 13, 14, 15, 16, 17]. These disturbances can be deterministic or stochastic and can affect different components of the system, for example, the system's dynamic, the control operator, the initial state..., which can drive the system to unstable behavior, or constraints violations. In order to contribute in this thematic, an application of the proposed approach to a class of perturbed systems is also considered.

Most of these researches are limited to the continuous case, and the results are based on the assumption that system states must be fully accessible [18], whereas in practice, this assumption is often unreasonable. In practice, the state is not often available (For instance, unknown disturbances infecting the initial state leads to unknown states). Therefore, it is necessary to estimate this unmeasured state 
vector. Then, we consider also a robust tracking problem for a class of discrete-time linear systems with inaccessible state.

The rest of the manuscript is organized as follows: In Section II, the model following problem to be tackled is stated and some standard assumptions are introduced, with the main theoretical results. In Section III, an application of the developed approach to a class of disturbed systems is proposed. In Section IV, a numerical example is given to illustrate the use of our results. The case of linear systems with inaccessible states is considered in Section V. The paper is concluded in Section VI.

\section{PROBLEM STATEMENT AND SOME PRELIMINARIES}

Consider the linear, controlled, discrete-time system described by

$$
\left\{\begin{array}{c}
x_{i+1}=A x_{i}+B u_{i} \\
x_{0} \in \mathbb{R}^{n}
\end{array}\right.
$$

and the associated output function is :

$$
y_{i}=C x_{i} \in \mathbb{R}^{p}
$$

where the state variable $x_{i} \in \mathbb{R}^{n}$ and $A, B, C$ are respectively $(n \times n),(n \times m),(p \times n)$ matrices. $u_{i} \in \mathbb{R}^{m}$ is the control function, which is introduced such that the associated output function (2) tracks a desired output $y_{i}^{m}$ generated by a reference system of the form

$$
\left\{\begin{array}{c}
x_{i+1}^{m}=A_{m} x_{i}^{m} \\
y_{i}^{m}=C_{m} x_{i}^{m}
\end{array}\right.
$$

where $x_{i}^{m}$ is the state vector of the reference model, and $y_{i}^{m} \in \mathbb{R}^{p}$ has the same dimension as $y_{i}$. As pointed out in [1], not all models of the form given in (3) can be tracked by a system given in (1) with a feedback controller.

\subsection{Case 1: Systems with accessible states}

In this subsection, we assume that the system states are fully accessibles, and we introduce for (1) the following standard assumption

Assumption 1 . The pair $(A, B)$ given in 11 is completely controllable.

It follows from Assumption 1 that there exists an $(m \times n)$ constant matrix $K$ such that $A+B K$ is Hurwitz. And for any given symmetric positive definite matrix $Q$, there exists an unique symmetric positive definite matrix $P$ as the solution of the Lyapunov equation

$$
P=(A+B K)^{T} P(A+B K)+Q
$$

In this work, the requirement for the developed controller to force the system output to follow the reference output model (3) as closely as possible is the following assumption.

Assumption 2 . There exist matrices $R, G, G_{e}$ and $H$ given by

$$
\begin{aligned}
G & =R^{T} \times\left[R R^{T}\right]^{-1} C_{m} \\
R & =C(A+B K)^{-1} B K \\
G_{e} & =(A+B K)^{-1} B K G \\
H & =B^{T}\left[B B^{T}\right]^{-1} G_{e} A_{m}
\end{aligned}
$$

Where $K$ is the above motioned matrix. If one of these matrices cannot be found, a different model must be chosen.

The output tracking error $e_{i}$ and a new auxiliary state vector $\widetilde{x}_{i}$ are defined as

$$
\begin{aligned}
\widetilde{x}_{i} & =x_{i}-G_{e} x_{i}^{m} \\
e_{i} & =y_{i}-y_{i}^{m}
\end{aligned}
$$

Where $G_{e}$ is defined in (7). From (3), (7), 9) and (10), one can obtain

$$
e_{i}=y_{i}-y_{i}^{m}=C \tilde{x_{i}}
$$

It follows from (11) that

$$
\left\|e_{i}\right\| \leq\|C\|\left\|\tilde{x}_{i}\right\|
$$

Since $\|C\| \leq \infty$, one can conclude that the convergence of $\widetilde{x}_{i}$ to the origin is sufficient for the tracking goal.

In this paper we propose a feedback control law described as follows

$$
u_{i}=K x_{i}+(H-K G) x_{i}^{m}
$$

Where $G$ and $H$ are defined in (5) and (8) respectively.

Theorem 1. Suppose that Assumptions 1 and 2 are satisfied. Then the control law (13) drives the system output (2) to asymptotically track the output of the reference system (3).

Proof. It follows from (5), (7), 8) and (9) that

$$
\begin{aligned}
\widetilde{x}_{i+1}= & x_{i+1}-G_{e} x_{i+1}^{m} \\
= & A x_{i}+B K \widetilde{x}_{i}+B K G_{e} x_{i}^{m} \\
& +B(H-K G) x_{i}^{m}-G_{e} A_{m} x_{i}^{m} \\
\widetilde{x}_{i+1}= & (A+B K) \widetilde{x}_{i}
\end{aligned}
$$

Constructing now the Lyapunov function as

$$
V\left(x_{i}\right)=x_{i}^{T} P x_{i}
$$

where $P$ is the unique solution of Lyapunov equation (4). The increment of the Lyapunov function in 15 is given by

$$
\begin{aligned}
\nabla V\left(\widetilde{x}_{i+1}\right) & =\widetilde{x}_{i+1}^{T} P \widetilde{x}_{i+1}-\widetilde{x}_{i}^{T} P \widetilde{x}_{i} \\
& =\widetilde{x}_{i}^{T}(A+B K)^{T} P(A+B K) \widetilde{x}_{i}-\widetilde{x}_{i}^{T} P \widetilde{x}_{i} \\
& =-\widetilde{x}_{i}^{T} Q \widetilde{x}_{i} \leq 0
\end{aligned}
$$

This shows that all trajectories of the closed-loop system (14) will converge to the origin. Then it can be obtained from (12) that the tracking error $e_{i}$ decreases asymptotically towards zero. This completes the proof.

Remark. Note that the result of theorem 2.1 is satisfied for all $x_{0} \in$ $\mathbb{R}^{n}$.

\subsection{Case 2: Systems with inaccessible states}

In this subsection, we assume that the state variable is unknown, thus, an observer is designed firstly, and then based on the observed states, the controller that guarantees the tracking goal, is designed. Consider the linear, controlled, discrete-time system described by

$$
\left\{\begin{array}{c}
x_{i+1}^{p}=A x_{i}^{p}+B u_{i} \\
x_{0}^{p} \in \mathbb{R}^{n}
\end{array}\right.
$$

and the associated output function is :

$$
y_{i}^{p}=C x_{i}^{p} \in \mathbb{R}^{p}
$$

where the state variable $x_{i}^{p} \in \mathbb{R}^{n}$ and $A, B, C$ are respectively $(n \times n),(n \times m),(p \times n)$ matrices. 
Based on the fact that the output function $y_{i}^{p}$ is measurable, an observer is introduced to estimate the state variable $x_{i}^{p}$ as follows

$$
\left\{\begin{array}{c}
z_{i+1}=F z_{i}+L y_{i}^{p}+B u_{i} \\
z_{0} \in \mathbb{R}^{n}
\end{array}\right.
$$

Where $F$ and $L$ are constant matrices with appropriate dimension satisfying

$$
\begin{aligned}
F & \text { is asymptotically stable } \\
F+L C & =A
\end{aligned}
$$

Let's define the observation error $\tilde{e}_{i}$ as follows

$$
\tilde{e}_{i}=z_{i}-x_{i}^{p}
$$

Thus we have

$$
\begin{aligned}
\tilde{e}_{i+1} & =F z_{i}+L C x_{i}^{p}+B u_{i}-A x_{i}^{p}-B u_{i} \\
& =F \tilde{e}_{i}+(F+L C-A) x_{i}^{p}
\end{aligned}
$$

By using 20, we have

$$
\tilde{e}_{i+1}=F \tilde{e}_{i}
$$

It is deduced from (19) that $\tilde{e}_{i} \rightarrow 0$, which proves the observation goal, and

$$
\left\|y_{i}^{p}-C z_{i}\right\| \rightarrow 0
$$

In the case where the system is autonomous (uninfected), this reduces to

$$
\left\{\begin{array}{c}
x_{i+1}^{m}=A_{m} x_{i}^{m} \\
y_{i}^{m}=C_{m} x_{i}^{m} \in \mathbb{R}^{n}
\end{array}\right.
$$

Based on results of section 2, a control law $u_{i}$ is designed for (16) such that the associated output function (17) tracks the desired output generated by the reference system 3 . The control law in (13) cannot be used here because the state variable is not available, hence the importance of the observer $(18)$.

The proposed control law is

$$
u_{i}=K z_{i}+(H-K G) x_{i}^{m}-M L y_{i}^{p}
$$

Where

$$
\begin{aligned}
G & =R^{T} \times\left[R R^{T}\right]^{-1} C_{m} \\
R & =C(F+B K)^{-1} B K \\
G_{e} & =(F+B K)^{-1} B K G \\
M & =B^{T}\left[B B^{T}\right]^{-1} \\
H & =M G_{e} A_{m}
\end{aligned}
$$

and $K$ is a constant matrix chosen in the way that $(F+B K)$ is Hurwitz invertible matrix. If one of these matrices cannot be found, a different model must be chosen.

Let's define an auxiliary variable as follows

$$
\tilde{x}_{i}=z_{i}-G_{e} x_{i}^{m}
$$

Where $G_{e}$ is given by 26 .

$$
\begin{aligned}
\left\|C z_{i}-C_{m} x_{i}^{m}\right\| & =\left\|C\left(z_{i}-G_{e} x_{i}^{m}\right)\right\| \\
& \leq\|C\|\left\|\tilde{x}_{i}\right\|
\end{aligned}
$$

By passing to the limit, and by 21 it is deduced that

$$
\left\|y_{i}^{p}-y_{i}^{m}\right\| \leq\|C\|\left\|\tilde{x}_{i}\right\|
$$

It is clear from 30 that the convergence of $\tilde{x}_{i}$ to the origin, is sufficient to achieve the tracking goal. Then we have the following result.

Theorem 2. If matrices (2428) exist, then the control law 23 drives the output function (17) to asymptotically track the output of the reference system (3).

Proof. It follows from 24, 26, 28, and 29, that

$$
\begin{aligned}
\widetilde{x}_{i+1}= & z_{i+1}-G_{e} x_{i+1}^{m} \\
= & F z_{i}+L y_{i}^{p}+B K z_{i}+B(H-K G) x_{i}^{m} \\
& -L y_{i}^{p}-G_{e} A_{m} x_{i}^{m} \\
= & F \tilde{x}_{i}+F G_{e} x_{i}^{m}+B K G_{e} x_{i}^{m}-B K G_{e} x_{i}^{m} \\
& +B K z_{i}-B K G x_{i}^{m} \\
\widetilde{x}_{i+1}= & (F+B K) \widetilde{x}_{i}
\end{aligned}
$$

Constructing now the Lyapunov function as

$$
V\left(x_{i}\right)=x_{i}^{T} P x_{i}
$$

where $P$ is the unique solution of Lyapunov equation

$$
P=(F+B K)^{T} P(F+B K)+Q
$$

for a given symmetric positive definite matrix $Q$. The increment of the Lyapunov function in 32 is given by

$$
\begin{aligned}
\nabla V\left(\widetilde{x}_{i+1}\right) & =\widetilde{x}_{i+1}^{T} P \widetilde{x}_{i+1}-\widetilde{x}_{i}^{T} P \widetilde{x}_{i} \\
& =\widetilde{x}_{i}^{T}(F+B K)^{T} P(F+B K) \widetilde{x}_{i}-\widetilde{x}_{i}^{T} P \widetilde{x}_{i} \\
& =-\widetilde{x}_{i}^{T} Q \widetilde{x}_{i} \leq 0
\end{aligned}
$$

This shows that all trajectories of the closed-loop system 31 will converge to the origin. Then it can be obtained from (12) that the tracking error $e_{i}$ decreases asymptotically towards zero. This completes the proof.

\section{APPLICATION TO A SENSITIVITY PROBLEM}

Consider the linear, controlled, discrete-time system described by

$$
\left\{\begin{array}{l}
x_{i+1}^{p}=A x_{i}^{p}+B u_{i} \\
x_{0}^{p}=\alpha x_{0}+\beta \in \mathbb{R}^{n}
\end{array}\right.
$$

and the associated output function is :

$$
y_{i}^{p}=C x_{i}^{p} \in \mathbb{R}^{p}
$$

where the state variable $x_{i}^{p} \in \mathbb{R}^{n}$ and $A, B, C$ are respectively $(n \times n),(n \times m),(p \times n)$ matrices, and $\beta \in \mathbb{R}^{n}$ and $\alpha \in \mathbb{R}$ are disturbances that infect the initial state, knowing that they are supposed inevitable. In the case where the system is autonomous (uninfected), this reduces to

$$
\left\{\begin{array}{c}
x_{i+1}^{m}=A_{m} x_{i}^{m} \\
y_{i}^{m} \stackrel{=}{=} C_{m} x_{i}^{m} \in \mathbb{R}^{n}
\end{array}\right.
$$

We introduce the control law $u_{i}$ in (34) such that the associated output function (35) tracks the desired output generated by the reference (uninfected) system 36 .

Definition. For a given $\epsilon>0$, and $T \in \mathbb{N}^{*}$, a disturbance $(\alpha, \beta) \in \mathbb{R} \times \mathbb{R}^{n}$ is said to be $\epsilon_{T}$-tolerable if the corresponding output function $y_{i}^{p}$ satisfies

$$
\left\|y_{i}^{p}-y_{i}^{m}\right\| \leq \epsilon, \forall i \geq T
$$

where $y_{i}^{m}$ is the output function of the reference (uninfected) system. 
Theorem 3. Given $\epsilon>0, T \in \mathbb{N}^{*}$ and a disturbance $(\alpha, \beta) \in \mathbb{R} \times \mathbb{R}^{n}$. Suppose that Assumptions 1 and 2 are satisfied. Then, there exists a control law $u_{i}$ that makes the disturbance $(\alpha, \beta) \epsilon_{T}$-tolerable.

Proof. Given an $\epsilon>0, T \in \mathbb{N}^{*}$. It is clear that

$$
\left\|C \tilde{x_{i}}\right\| \leq\|C\|\left\|\tilde{x_{i}}\right\|
$$

By Theorem 2.1 remark 2.1 assumption 1 and 2 and (14) there exists a matrix $K$ such that the corresponding control law given by (13) ensures that

$$
\left\|\widetilde{x}_{i}\right\| \leq \frac{\epsilon}{\|C\|}, \forall i \geq T
$$

Which implies, from (37), that

$$
\left\|C \widetilde{x}_{i}\right\| \leq \epsilon, \forall i \geq T
$$

Then, it follows from (11) that

$$
\left\|y_{i}^{p}-y_{i}^{m}\right\| \leq \epsilon, \forall i \geq T
$$

Which means that the disturbance associated to $y_{i}^{p}$ is $\epsilon_{T}$-tolerable.

\section{ILLUSTRATIVE EXAMPLES}

\section{Example 1}

To illustrate the utilization of our approach, in this subsection, we consider the following numerical example. Here, a linear discretetime system is given as follows:

$$
\left\{\begin{array}{c}
x_{i+1}^{p}=A x_{i}^{p}+B u_{i} \\
y_{i}^{p}=C x_{i}^{p} \\
x_{0}^{p}=\alpha x_{0}+\beta \in \mathbb{R}^{2}
\end{array} i \geq 0\right.
$$

where

Table 1.

Matrices

\begin{tabular}{|c|c|c|c|}
\hline A & B & C & $x_{0}$ \\
\hline \hline$\left(\begin{array}{cc}1.5 & -3 \\
0 & -2\end{array}\right)$ & $\left(\begin{array}{ll}2 & -2 \\
6 & -3\end{array}\right)$ & $\left(\begin{array}{ll}0.9 & 1.3\end{array}\right)$ & $\left(\begin{array}{ll}0.1 & 1\end{array}\right)^{T}$ \\
\hline
\end{tabular}

and the perturbation $\alpha=2$ and $\beta=(-0.4,-1.1)^{T}$. The control input $u_{i}$ is used in order to $y_{i}^{p}$ tracks the output response of the reference (uninfected) system given by

$$
\left\{\begin{aligned}
x_{i+1}^{m} & =A_{m} x_{i}^{m} \in \mathbb{R}^{2} \\
y_{i}^{m} & =C_{m} x_{i}^{m} \in \mathbb{R}
\end{aligned}\right.
$$

where $A_{m}=\left(\begin{array}{cc}0.9 & 2 \\ 0 & 0.9\end{array}\right), C_{m}=\left(\begin{array}{ll}1 & 0.2\end{array}\right), x_{0}^{m}=\left(\begin{array}{ll}1 & 0.1\end{array}\right)^{T}$. It's clear that the pair $(A, B)$ is controllable, then we choose $K$ such that

$$
K=\left(\begin{array}{cc}
1.15 & -0.6 \\
2.3 & -2.1
\end{array}\right) \text { and } A+B K=\left(\begin{array}{cc}
-0.8 & 0 \\
0 & 0.7
\end{array}\right)
$$

Matrices (5), (7) and (8) are given, respectively, by

$$
\begin{aligned}
G & =\left(\begin{array}{ll}
0.2758 & 0.0552 \\
0.1747 & 0.0349
\end{array}\right), G_{e}=\left(\begin{array}{ll}
0.1377 & 0.0275 \\
0.6739 & 0.1348
\end{array}\right), \\
H & =\left(\begin{array}{ll}
0.1402 & 0.3396 \\
0.0783 & 0.1896
\end{array}\right)
\end{aligned}
$$
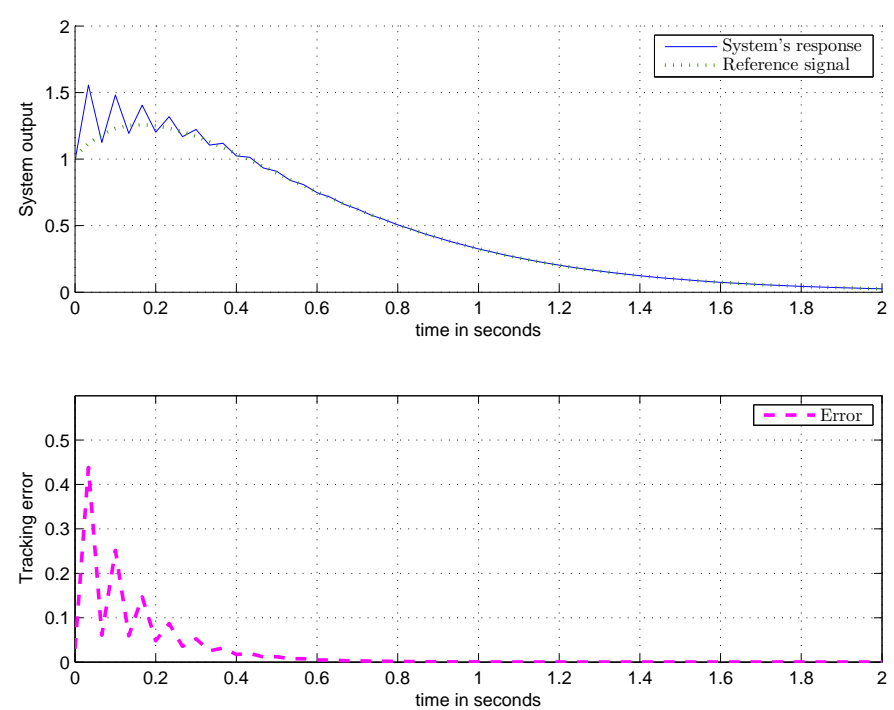

Fig. 1. Tracking performance and Tracking error
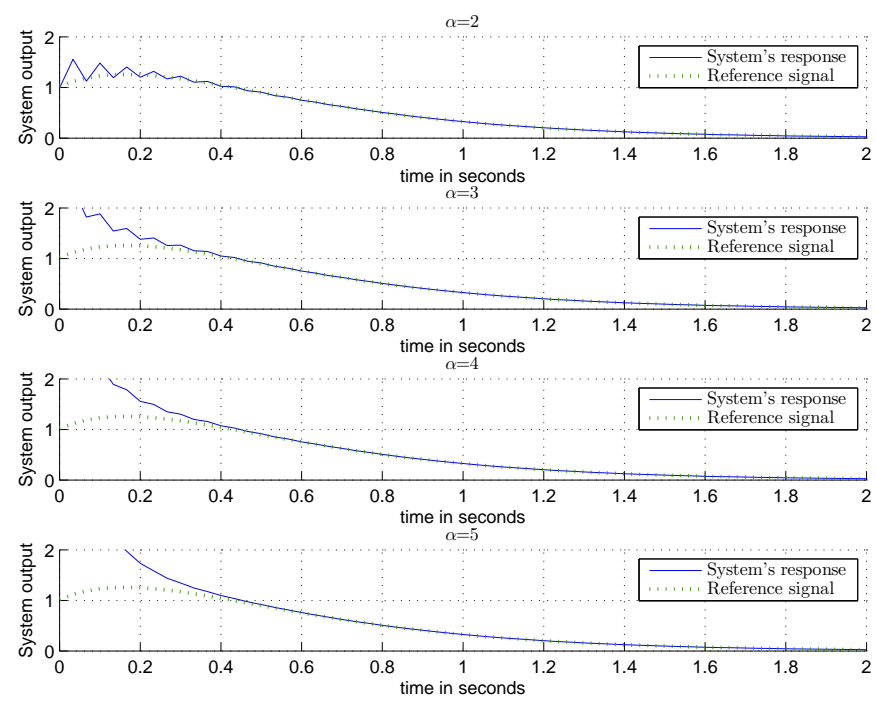

Fig. 2. Impact of disturbances $\alpha$ on tracking performance where $\beta=$ $(-0.4,-1.1)$.

From Figure 1, we can conclude that with the chosen $K$, the associated control law $u_{i}$ ( given in (13), makes the disturbance $(\alpha, \beta)$ for this example, $0.1_{1}$-tolerable.

Comment. 1) In this example, we should note that the disturbance $(\alpha, \beta)$ is arbitrary chosen.

2) Note that the reference system and the nominal system have the same dimension. Thus, to show the effectiveness of our control design, a three dimensional reference system is tracked by a two dimensional system in the following example. 

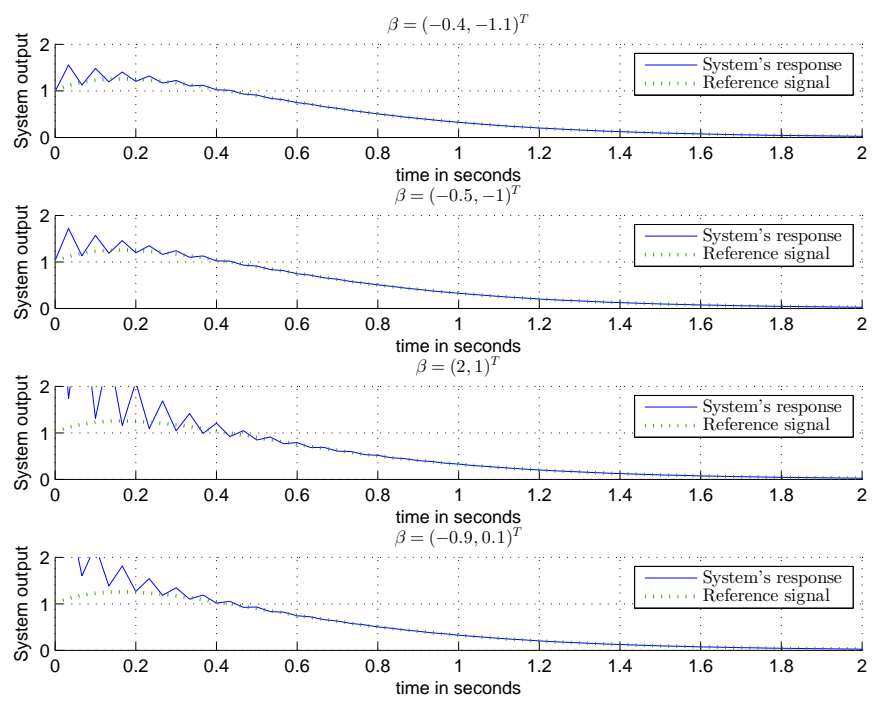

Fig. 3. Impact of disturbances $\beta$ on tracking performance where $\alpha=2$.

\section{Example 2}

In this subsection, we consider the following numerical example

$$
\left\{\begin{array}{c}
x_{i+1}=A x_{i}+B u_{i} \\
y_{i}=C x_{i} \in \mathbb{R} \quad i \geq 0 \\
x_{0} \in \mathbb{R}^{2}
\end{array}\right.
$$

where $A, B, C$ and $x_{0}$ are given in table 1 In this example we consider that the reference system does not have the same dimension of the system (39), given by

$$
\left\{\begin{aligned}
x_{i+1}^{m} & =A_{m} x_{i}^{m} \\
y_{i}^{m} & =C_{m} x_{i}^{m} \in \mathbb{R}
\end{aligned}\right.
$$

where $A_{m}=\left(\begin{array}{ccc}0.8 & 1.2 & -1 \\ 0 & 0.7 & 1 \\ 0 & 0 & 0.5\end{array}\right), C_{m}=\left(\begin{array}{lll}1 & 0.2 & 0.5\end{array}\right)$ and $x_{0}^{m}=$

$\left(\begin{array}{lll}0 & 1 & 0.1\end{array}\right)^{T}$. By using the same matrix $K$ given in 38 , matrices (5), (7) and (8) are given, respectively, by

$$
\begin{gathered}
G=\left(\begin{array}{ccc}
0.2758 & 0.0552 & 0.1379 \\
0.1747 & 0.0349 & -0.0874
\end{array}\right), \\
G_{e}=\left(\begin{array}{lll}
0.1377 & 0.0275 & 0.0688 \\
0.6739 & 0.1348 & 0.3370
\end{array}\right), \\
H=\left(\begin{array}{lll}
0.1246 & 0.2088 & -0.0857 \\
0.0696 & 0.1165 & -0.0478
\end{array}\right)
\end{gathered}
$$

By using the control law (13), figure 3 shows that the tracking error decreases asymptotically to zero, and the output of the system (39) tracks the reference output of the system (40).

Remark. Note that the above results are based on the assumption that system states must be fully accessible, whereas in practice, this assumption is often unreasonable. This has motivated us to improve our results by using an observer-based control for discrete-time linear systems with inaccessible state.

\section{CONCLUSION}

The problem of robust tracking and model following for a class of linear discrete-time systems has been considered. Based on the
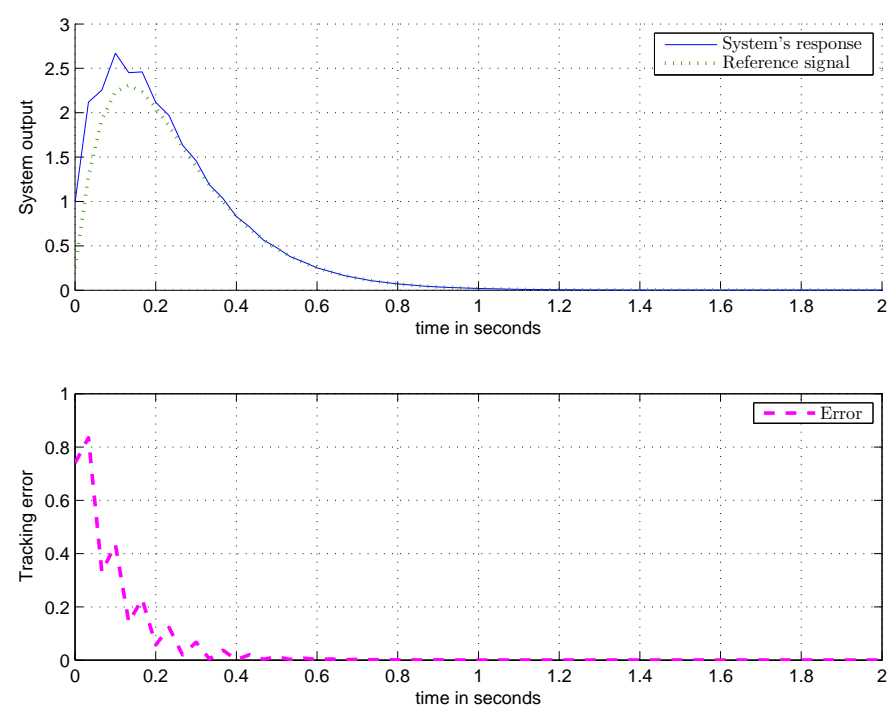

Fig. 4. Tracking performance and Tracking error corresponding to example 2

solution of the Lyapunov equation, we have shown that by employing the proposed adaptive robust tracking controller, the tracking error can be guaranteed to decrease asymptotically to zero. An application of the proposed approach for a class of disturbed systems is also considered. Illustrative examples have been provided to demonstrate the effectiveness of this control technique. By assuming that the system states are not fully accessibles, an observerbased controller is designed such that the tracking error converges asymptotically towards zero.

\section{REFERENCES}

[1] Hopp, T. H., \& Schmitendorf, W. E. (1990). Design of a linear controller for robust tracking and model following. Journal of dynamic systems, measurement, and control, 112(4), 552558.

[2] Oucheriah, S. (1999). Robust tracking and model following of uncertain dynamic delay systems by memoryless linear controllers. Automatic Control, IEEE Transactions on, 44(7), 1473-1477.

[3] Lawrence, D. A., \& Rugh, W. J. (1995). Gain scheduling dynamic linear controllers for a nonlinear plant. Automatica, 31(3), 381-390.

[4] Ni, M., \& Li, G. (2006). A direct approach to the design of robust tracking controllers for uncertain delay systems. Asian Journal of Control, 8(4), 412.

[5] Ni, M. L., Er, M. J., Leithead, W. E., \& Leith, D. J. (2001). New approach to the design of robust tracking and model following controllers for uncertain delay systems. IEE Proceedings-Control Theory and Applications, 148(6), 472477.

[6] Basher, A. H., Mukundan, R., \& O'CONNOR, D. A. (1986) Memoryless feedback control in uncertain dynamic delay systems. International Journal of Systems Science, 17(3), 409415.

[7] Shyu, K. K., \& Chen, Y. C. (1995). Robust tracking and model following for uncertain time-delay systems. International Journal of Control, 62(3), 589-600. 
[8] Corless, M., Leitmann, G., \& Ryan, E. P. (1984, September). Tracking in the presence of bounded uncertainties. In 4th Int. Conf. Control Theory, Cambridge, UK.

[9] Corless, M., Goodall, D. P., Leitmann, G., \& Ryan, E. P. (1985). Model-following controls for a class of uncertain dynamical systems. In Proceedings of the 7th IFAC Symposium on Identification and System Parameter Estimation, York University, York, England.

[10] Wu, H. S. (2000). Robust tracking and model following control with zero tracking error for uncertain dynamical systems. Journal of Optimization Theory and Applications, 107(1), 169-182.

[11] Wu, H. (2004). Adaptive robust tracking and model following of uncertain dynamical systems with multiple time delays. Automatic Control, IEEE Transactions on, 49(4), 611-616.

[12] Fridman, E. (2002). Effects of small delays on stability of singularly perturbed systems. Automatica, 38(5), 897-902.

[13] P. (1997). Stability of perturbed systems with time-varying delays. Systems \& Control Letters, 31(3), 155-163.

[14] Floquet, T., Barbot, J. P., \& Perruquetti, W. (2003). Higherorder sliding mode stabilization for a class of nonholonomic perturbed systems. Automatica, 39(6), 1077-1083.

[15] Assawinchaichote, W., \& Nguang, S. K. (2004). H? filtering for fuzzy singularly perturbed systems with pole placement constraints: an LMI approach. Signal Processing, IEEE Transactions on, 52(6), 1659-1667.

[16] Chen, W., Jiao, L., Li, R., \& Li, J. (2010). Adaptive backstepping fuzzy control for nonlinearly parameterized systems with periodic disturbances. Fuzzy Systems, IEEE Transactions on, 18(4), 674-685.

[17] Zakary, O., \& Rachik, M. (2016). The \$ epsilon \$-capacity of a gain matrix and tolerable disturbances: Discrete-time perturbed linear systems. arXiv preprint arXiv:1608.00426.

[18] Zakary, O., \& Rachik, M. (2016). Applied Lyapunov Stability on Output Tracking Problem for a Class of Discrete-Time Linear Systems. arXiv preprint arXiv:1607.02744. 